\title{
Erratum: Statistics of chaotic resonances in an optical microcavity [Phys. Rev. E 93, 040201(R) (2016)]
}

Li Wang, Domenico Lippolis, Ze-Yang Li, Xue-Feng Jiang, Qihuang Gong, and Yun-Feng Xiao

(Received 25 July 2016; published 5 August 2016)

DOI: 10.1103/PhysRevE.94.029902

Equation (3) in the paper should read

$$
\left|a_{\omega}\right|^{2}=\epsilon^{2} \frac{n_{\gamma}^{2}}{\left(\Gamma+n_{\gamma}\right)^{2}} .
$$

The denominator was incorrectly stated as $\Gamma^{2}+n_{\gamma}^{2}$. This correction affects the graphs of the probability of excitation of regular modes in Fig. 2, which we replot below, showing no qualitative changes from the original publication.

The fittings to the experimental data, portrayed in Fig. 4, are also reproduced here.

We also provide corrections for the fitting parameters and the $\chi^{2}$, stated here for deformation factors $\eta \simeq 11.7 \%$ and $6 \%$, respectively: the RMT expression yields $\gamma \simeq 0.17,0.16$, and $\chi^{2} \simeq 2.5$ and 5; the semiclassical prediction instead produces $\chi^{2} \simeq 1.2$ and 1

The semiclassical theory still captures the experimental data better than the purely RMT-based prediction, the difference being even more marked here than in the original paper (Fig. 4). Our conclusions are therefore not only unchanged, but rather strengthened.
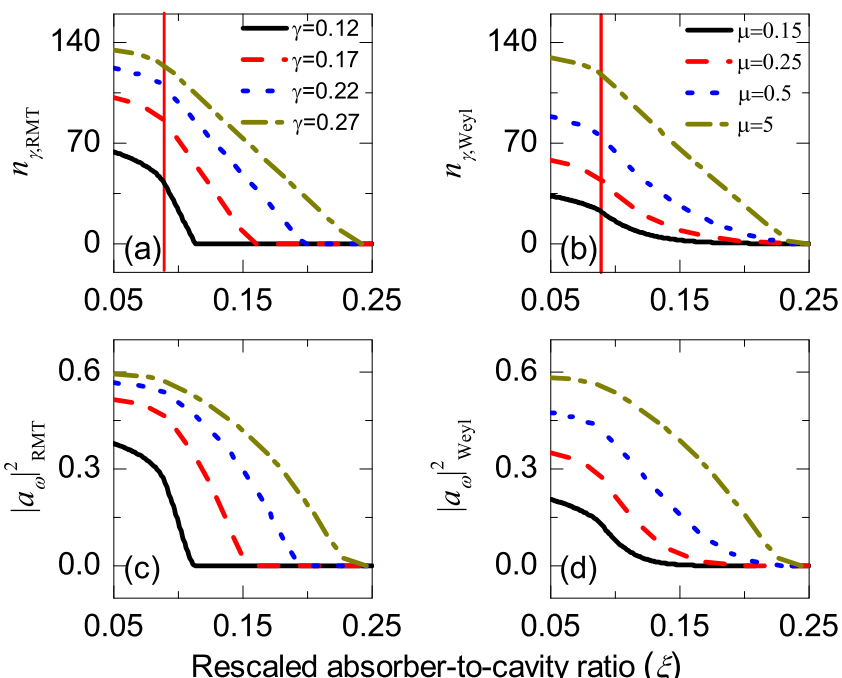

FIG. 2. (a), (b) Number of chaotic states $n_{\gamma}$ vs the rescaled absorber-to-cavity ratio $\xi$, obtained by RMT and Weyl law, respectively. (c), (d) The corresponding expectations for $\left|a_{\omega}\right|^{2}$. In (d), the smaller $\mu$, the more visible the tail of the excitation probability. The red vertical line corresponds to the critical angle, $\sin \theta_{c} \simeq 0.69$. Here $\sin \theta_{\mathrm{th}}=0.6$.

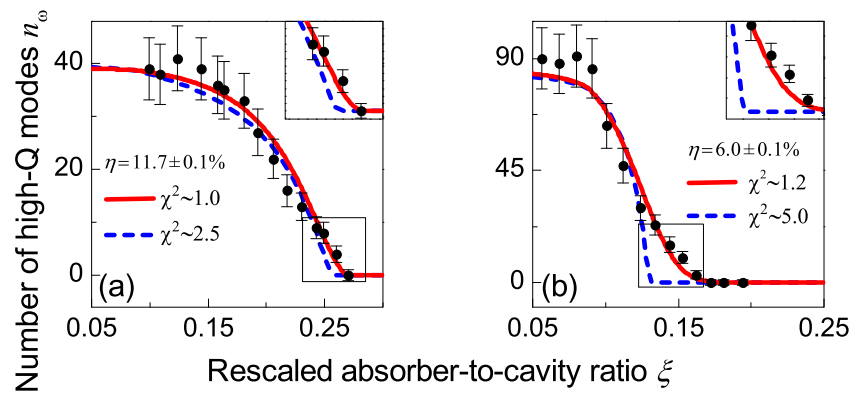

FIG. 4. (a), (b) Number of high- $Q$ regular modes $\left(n_{\omega}\right)$ observed experimentally in the microcavities of $\eta=11.7 \%$ and $6 \%$, respectively, vs rescaled absorber-to-cavity ratio $\xi$. Blue dashed and red solid curves are, respectively, RMT and Weyl-law best fits, whose goodness is assessed by $\chi^{2}$. Here $\sin \theta_{c} \simeq 0.69$ and $\sin \theta_{\text {th }}=0.6$. Inset: area where the two curves differ most. 